Ingreso de lecturas de consumo de agua potable en EMAPAL-Azogues, a través de dispositivos móviles.

Juan Pablo Cuenca Tapia, Juan Carlos Ortega Castro

Recibido: marzo 2017

Aprobado: mayo 2017 


\title{
Ingreso de lecturas de consumo de agua potable en EMAPAL-Azogues, a través de dispositivos móviles
}

\section{Input of potable water consumption readings in EMAPAL-Azogues, through mobile devices}

\author{
Juan Pablo Cuenca Tapia. Juan Carlos Ortega Castro. \\ Unidad Académica de Ingeniería, Industria y Construcción \\ Universidad Católica de Cuenca. \\ Av. de las Américas y Tarqui. \\ Cuenca - Ecuador \\ jcuenca@ucacue.edu.ec
}

\begin{abstract}
Resumen
El presente artículo tiene como objetivo mejorar el registro de las lecturas de consumo del líquido vital sobre los medidores domiciliarios de los usuarios de la Empresa de Agua Potable y Alcantarillado de AzoguesEMAPAL, mediante una aplicación tecnológica, la misma que brindará a los lectores/digitadores, mejores condiciones de trabajo, permitiendo de esta manera la actualización inmediata de los datos. La funcionalidad de la aplicación permite que, gracias al creciente uso de sistemas de comunicación móviles y su ayuda a la interrelación entre personas, empresas y proyectos, el lector ingrese mediante el dispositivo móvil, SmartPhone o una Tablet con sistema operativo Android, a la aplicación vinculada a la base de datos, el cual digitará la lectura actual del medidor en la aplicación y automáticamente actualizará la base de datos, considerando que el tiempo estimado en digitalizar las lecturas en la base de datos es de 15 días aproximadamente, con la aplicación móvil se evita la re-digitalización y directamente se procede a realizar los reportes diarios de las lecturas realizadas para la elaboración de facturas.
\end{abstract}

Palabras clave: diagrama de caso, diagrama de clases, spinner, facturación, toma de lecturas, dispositivos móviles.

\begin{abstract}
This article is developed because EMAPAL-Azogues requires to improve the registration of the consumption of the vital liquid on the home meters of the users, through a technological application, which will provide the readers I digitizers better working conditions, allowing them the immediate updating of the data. The functionality of the application allows, thanks to the growing use of mobile communication systems and its help to the interrelation between people, companies and projects; The reader can enter via the mobile device, it can be a Smart Phone or a Tablet with Android operating system, the application linked to the database, which will enter the current meter reading in the application and automatically update the database, Considering that the estimated time to digitize the readings in the database is approximately 15 days, with the mobile application, a re-digitization is avoided and the daily reports of the readings made for the billing proces.
\end{abstract}

KEYWORDS: Case diagram, class diagram, spinner, billing, taking readings, mobile devices. 


\section{Introducción}

La Empresa Municipal de Agua Potable, Alcantarillado y Saneamiento de Azogues-EMAPAL, nace por la decisión mayoritaria del Ilustre Concejo Municipal en sesión efectuada el 30 de Marzo de 1974 mediante la expedición de la Ordenanza Nro. 13, iniciando sus actividades en forma absolutamente independiente el 1 de Agosto de 1974, mientras ejercía la Primera Dignidad de la Ciudad el Dr. Marco Romero Heredia; luego de haber estructurado el plano administrativo y de operación y recibiendo la transferencia de bienes y aportes económicos de capital. Originalmente el Directorio se integró por cinco Miembros: el alcalde, un edil, un ciudadano designado por el Concejo de fuera de su seno, un representante de los Colegios de Ingenieros Civiles, Arquitectos y de las Cámaras de Comercio y Agricultura, un representante del IEOS, y como secretario actuaba el Gerente ${ }^{1}$.

El departamento de Comercialización para realizar la facturación a los usuarios del servicio de agua potable, primeramente realiza la lectura mensual de consumo en los medidores ubicados en los domicilios de los abonados, se observa que las mediciones se realizan de manera manual a los 9776 usuario por parte de los lectores, los mismo que se distribuyen en 31 sectores registrando los resultados en las hojas de lectura del consumo de agua, no existe automatización en este proceso que facilite el trabajo, mejore el servicio a los usuarios y ahorren tiempo en la digitación a la base de datos, por lo expuesto es imperativa la necesidad del uso de tecnología mediante una aplicación móvil, que permita disminuir tiempo y costos, proporcionar innovación en el manejo de información y satisfacer la necesidad de manejar mayor cantidad de datos.

Los dispositivos móviles. - Constituyen cada vez más una realidad que ofrece al usuario, en un mismo y reducido aparato, funciones de comunicación y procesamiento de datos que van mucho más allá de las simples llamadas telefónicas o la ejecución de aplicaciones básicas. El desarrollo de aplicaciones web para móviles se ha incrementado con base a las necesidades de los usuarios ${ }^{2}$.

Los planes de desarrollo local y nacional, el cambio en la matriz productiva y energética del País y el Plan

\footnotetext{
${ }^{1}$ EMPRESA PÚBLICA MUNICIPAL DE AGUA POTABLE. (s.f.). Obtenido de

http://www.emapal.gob.ec/index.php?option=com_con tent $\&$ view $=$ article $\&$ id $=88 \&$ Itemid $=58$
}

2 Naj, N. A. (5 de junio de 2013). Attribution Noncommercial. Recuperado el 2 de marzo de 2014, de es.scribd.com: http://es.scribd.com/doc/145791290/MarcoTeorico\#scr ibd
Nacional del Buen Vivir, motivan la generación, búsqueda e investigación que permitan mejoras sociales en términos de conectividad, comunicación y servicio. Gracias a esto se puede fundamentar lo siguiente:

El objetivo 10 del Plan Nacional del Buen Vivir 2013 2017 establece impulsar la transformación de la matriz productiva y en su punto 10.2 considera "Articular la investigación científica, tecnológica y la educación superior con el sector productivo, para una mejora constante de la productividad y competitividad sistémica, en el marco de las necesidades actuales y futuras del sector productivo y el desarrollo de nuevos conocimientos. b. Tecnificar los encadenamientos productivos en la generación de materias primas y la producción bienes de capital, con mayor intensidad tecnológica en sus procesos productivos. c. Crear y fortalecer incentivos para fomentar la inversión privada local y extranjera que promueva la desagregación, transferencia tecnológica y la innovación. d. Implementar mecanismos de reactivación y utilización óptima de la capacidad instalada del Estado en actividades de producción y de generación de trabajo. e. Fomentar la sustitución selectiva de importaciones, considerando la innovación y tecnología como componentes fundamentales del proceso productivo, con visión de encadenamiento de industrias básicas e intermedias. f. Asegurar que los encadenamientos productivos de las industrias estratégicas claves, los sectores prioritarios industriales y de manufactura, generen desagregación y transferencia tecnológica en sus procesos productivos. g. Articular los programas de innovación participativa en el sector rural, en sistemas formales e informales, con acceso y uso de TIC para incrementar la cobertura de los servicios y fomentar el intercambio de conocimientos entre actores locales." 3 El objetivo 11 del Plan Nacional del Buen Vivir 2013 2017 por su parte establece el asegurar la soberanía y eficiencia de los sectores estratégicos para la transformación industrial y tecnológica y en su punto 11.1 "Reestructurar la matriz energética bajo criterios de transformación de la matriz productiva, inclusión, calidad, soberanía energética y sustentabilidad, con incremento de la participación de energía renovable." Siendo uno de sus principales enunciados el "Cuantificar el potencial de recursos de energías renovables para generación eléctrica." 5

\footnotetext{
${ }^{3}$ Secretaría Nacional de Planificación y Desarrollo (2013), Plan Nacional del Buen Vivir 2013 -2017, pág. 301

${ }^{4}$ Secretaría Nacional de Planificación y Desarrollo (2013), Plan Nacional del Buen Vivir 2013 -2017, pág. 322

5 ídem
} 
Estudios semejantes o información relevante permiten desarrollar el artículo de manera confiable en función de su factibilidad de ejecución, como lo expresan Puente y Robalino (2006), en su tesis: Sistema para la lectura de consumo de agua con dispositivos móviles. "El proyecto de titulación Sistema para la Lectura de Consumo de Agua con Dispositivos Móviles, está enfocado a la investigación de la tecnología móvil y su uso en una entidad pública. El sistema está diseñado para apoyar el proceso de toma de datos directamente en el lugar donde éstos se desarrollan a través del uso de dispositivos móviles". La aplicación del Proceso Unificado de Desarrollo hace posible la implementación del sistema detallando la arquitectura y las herramientas que han facilitado su construcción.

Otro caso como el de González y Sandino (2008), en su tesis: Estudios de protocolos entre PDA/PC y su aplicación en el Sistema de Facturación de la EAPA San Mateo, se concluye que "El estudio de los PDA's y los protocolos para la comunicación con el PC nos permite la extracción e ingreso de información al servidor de base de datos de la empresa, así como envía y recupera los datos de lecturas del Pocker PC, esto mediante la utilización del protocolo TCP/IP que puede efectuar interconexión entre redes alámbricas e inalámbricas pudiendo así utilizar Bluetooth o Wifi”.

El uso de dispositivos móviles o PDA's para el intercambio de información lo describen: Arturo Baz Alonso, Irene Ferreira Artime, María Álvarez Rodríguez, Rosana García Baniello, (2009), Universidad de Oviedo.'PDAs (Person al Digital Assintant), es una computadora de mano originalmente diseñada como agenda electrónica con un sistema de reconocimiento de escritura. Hoy día estos dispositivos, pueden realizar muchas de las funciones de una computadora de escritorio pero con la ventaja de ser portátil”.

\section{Materiales y Métodos}

Mediante la utilización de casos de uso se muestran el conjunto de requisitos de acuerdo con una función o tarea. En lugar de la tradicional lista de requisitos que quizá no trate de forma directa el uso de la solución, los casos de uso reúnen requisitos comunes basados en el tipo de función u objetivo. Los casos de uso definen qué harán los usuarios o funciones en la solución, los casos de uso de la aplicación para la toma de lecturas en los medidores de agua potable de los usuarios de EMAPAL-EP son los siguientes:

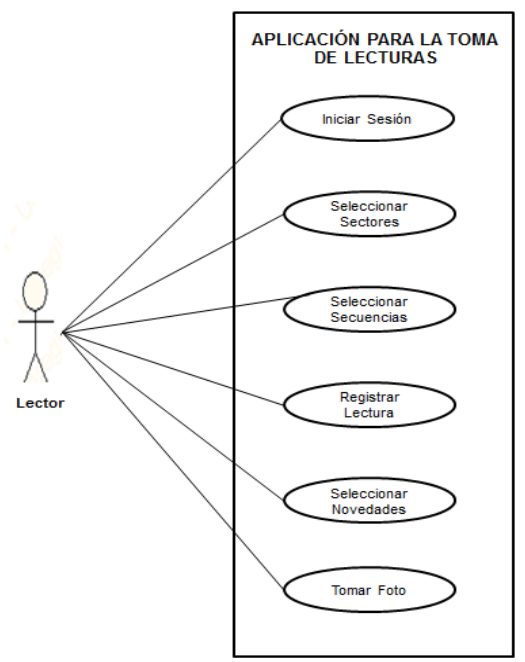

Figura 1. Diagrama de caso de USO

\section{ESPECIFICACIONES DE CASOS DE USO}

- CASO DE USO: "INICIAR SESIÓN"

Mediante este caso de uso el lector podrá ingresar a la aplicación mediante el inicio de sesión.
- CASO DE
USO:
"SELECCIONAR
SECTORES"

A través de este caso de uso el lector puede ingresar al sector que le haya sido asignado por el administrador.

\section{- CASO DE USO: "SELECCIONAR SECUENCIA"}

Este caso de uso contiene las secuencias que tiene cada uno de los diversos sectores que tiene dividida la empresa.

- CASO DE USO: "REGISTRAR LECTURA"

Permite ingresar la lectura de cada uno de los diversos medidores.

\section{- CASO DE USO: "SELECCIONAR NOVEDADES',}

Con este caso de uso el lector tendrá la opción de ingresar la novedad que se le presenta al momento de la toma de lectura.

\section{- CASO DE USO: "TOMAR FOTO"}

Con este caso de uso el lector podrá capturar la foto del medidor al cual le está tomando la lectura.

\section{DIAGRAMA DE CLASES}

Para visualizar las relaciones entre las clases involucradas en nuestro sistema presentamos la vista de diseño estático: 

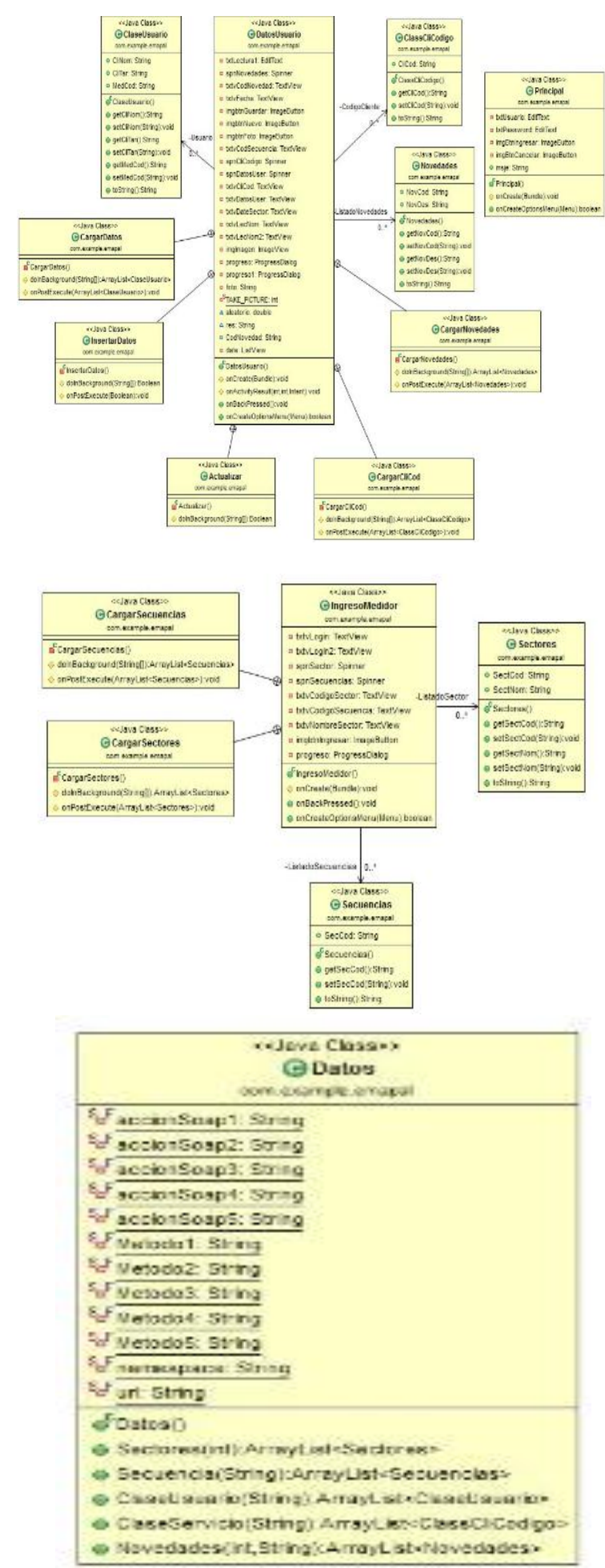

Figura 2. Diagrama de CLASES

\section{Resultados}

La aplicación permite ingresar a cada una de las pantallas en donde se encuentran los datos necesarios para que el lector se guie al momento de realizar la toma de lectura, la misma que se ingresara a la base de datos.

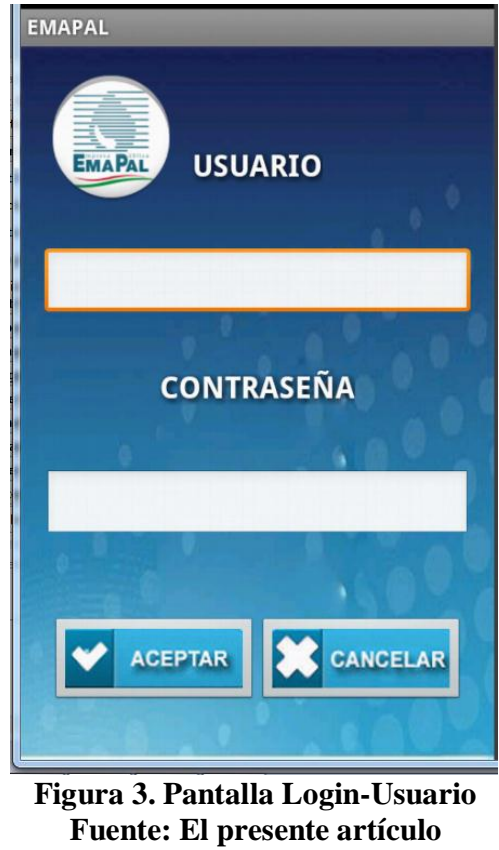

En la figura anterior se puede observar la pantalla principal Login-Usuario, en la que el lector podrá ingresar su usuario y contraseña para el inicio de sesión. Dicho usuario y contraseña es proporcionado por el administrador.

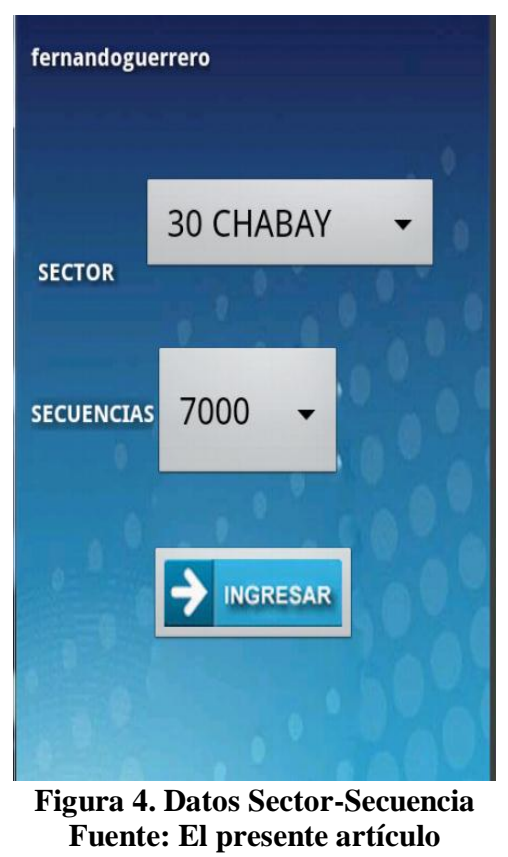

En la figura 4 se indica el nombre del lector que ingresa a la aplicación y la información de los sectores y secuencias donde se están tomando las lecturas. 


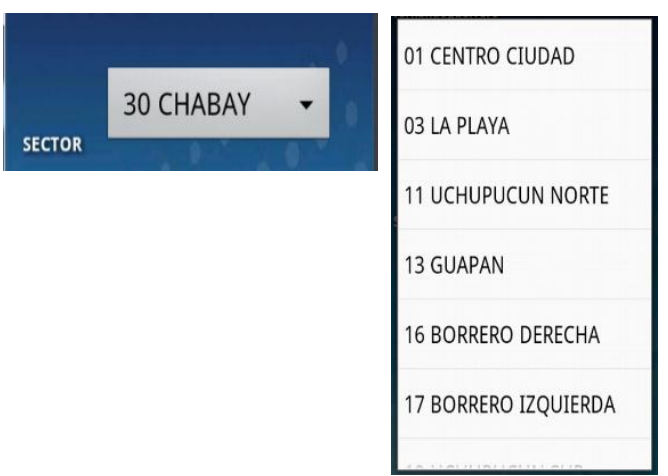

Figura 5. Spinner - Sector

Fuente: El presente artículo

Es necesaria la carga de cada uno de los sectores, con sus respectivos códigos y nombres, como es muestra en la figura 5.

La siguiente figura indica la carga de secuencias pertenecientes a los diferentes sectores de la ciudad.

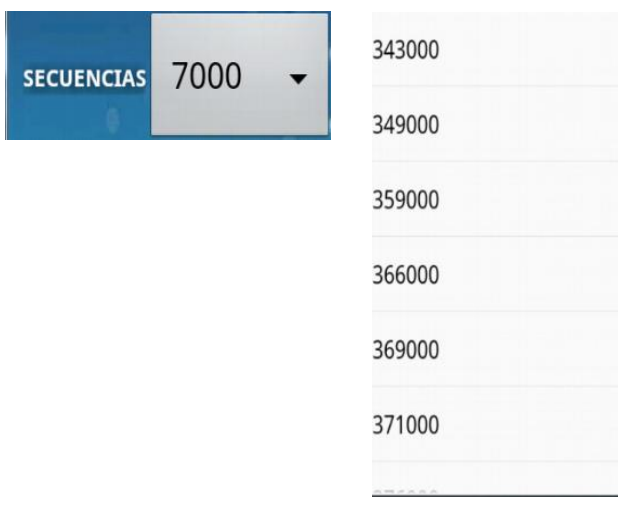

Figura 6. Spinner - Secuencias Fuente: El presente artículo

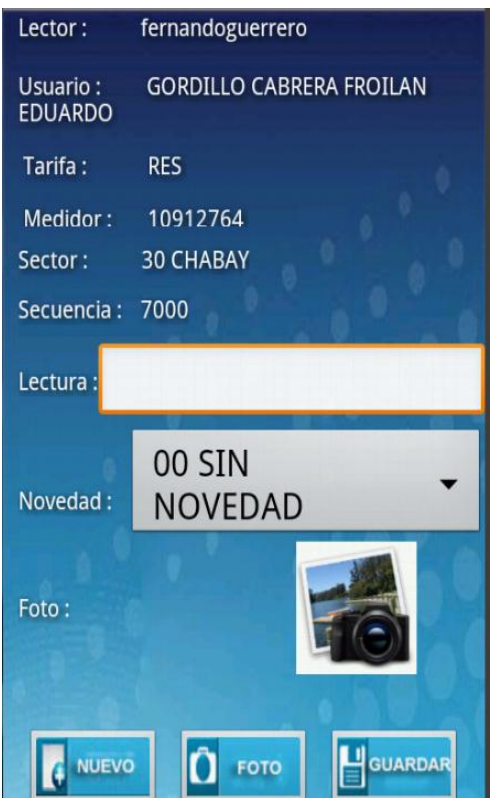

Figura 7. Datos - Usuario Fuente: El presente artículo
En la figura 7 se muestra la última pantalla de la aplicación donde se pueden visualizar los datos completos del usuario para que su lectura sea ingresada al sistema y se proceda a la respectiva facturación en las oficinas principales de EMAPAL.

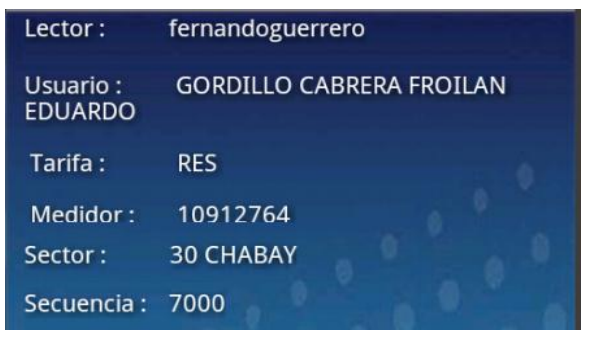

Figura 8. Datos

Fuente: El presente artículo

La figura 8 contiene la información relevante de la aplicación, la misma que se detalla de la siguiente manera:

Lector: Indica el nombre de la persona encargada de tomar la lectura.

Usuario: Contiene los nombres de personas y entidades los mismos que consumen el líquido vital.

Tarifa: Muestra si el usuario es residente, comercial, industriales, etc.

Medidor: Contiene el número de medidor perteneciente a cada usuario.

Sector y Secuencia: Es el número de sector y secuencia al cual pertenece al usuario.
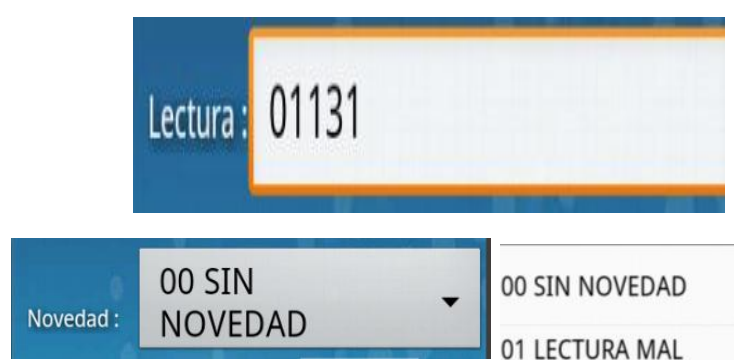

OO SIN NOVEDAD

01 LECTURA MAL REGISTRADA

02 CASA ABANDONADA

O3 PROMEDIO - MEDIDOR DAÑADO Y/O PARADO

04 SITIO

05 CONSUMOS ELEVADOS

Figura 9. Spinner - Novedad Fuente: El presente artículo

Una vez ingresados los datos, se procede a ingresar la lectura obtenida en la visita al domicilio, y el lector tendrá la opción de registra cualquier novedad que se presente con las diferentes alternativas que brinda la aplicación. Dichos inconvenientes presentados al momento de tomar la lectura se muestran en la figura 9 . 
Al finalizar la toma de lectura, registrar inconvenientes, tomar fotos de las evidencias necesarias, etc, se procede a guardar la información almacenada, la misma que irá a la base de datos creada para recibir los datos de cada lector en los diferentes sectores de la ciudad, y procesarlos en la institución.

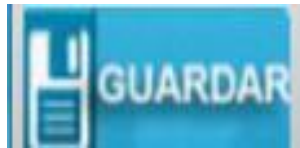

Figura 10. Botón Guardar

Fuente: El presente artículo

\section{CONCLUSIONES}

Al término del presente artículo se concluye:

- Que el uso de las tecnologías de la información y comunicación, permiten que mediante un sistema o equipo Android se obtengan beneficios en función de su desarrollo, aplicación, arquitectura, componentes y funcionalidad, para la mejora en procesos

\section{Referencias}

1. Bandara, L. (10 de abril de 2014). Zona de Fresnel. Obtenido de Mundo Telecomunicaciones: www.mundotelecomunicaciones 1.blogsp ot.com

2. http://biblioteca.epn.edu.ec/cgibin/kohalopacdetail.pl?biblionumber $=6145$

3. http://bibliotecas.espoch.edu.ec/cgibin/koha/opacdetail.pl? biblionumber $=42773$

4. Casanova, V. (28 de junio de 2012). ¿Qué es el Espectro Electromagnético? Obtenido de Astrofísica y Física: http://www.astrofisicayfisica.com

5. Cisco. (s.f.). Cisco Small Business 200 Series Smart Switches. Obtenido de Cisco 200 Series Switches: www.cisco.com

6. Commscope. (2015). Commscope. Obtenido de Sistemas de antenas: es.commscope.com

7. CONATEL. (2001). REGLAMENTO PARA LA PRESTACION DE SERVICIOS PORTADORES. Ecuador.

8. CONATEL. (2012). Plan Nacional de Frecuencias. Ecuador.

9. CONGRESO NACIONAL. (1992). LEY ESPECIAL cotidianos y toma de decisiones a tiempo a nivel empresarial.

- La aplicación contiene una interfaz amigable y fácil de ser manejada, de tal manera que cualquier usuario puede tener acceso a la misma y facilite su manejo, también permite que los datos se actualicen mediante procesos que evitan al usuario de la carga de actualizar manualmente la información.

- Se reemplazarán las hojas de lectura y el bolígrafo que utiliza el personal de la empresa para el registro manual de la lectura, por un registro online con el valor consumido mensualmente, permitiendo tener de inmediato los valores en la base de datos.

- Otro aspecto importante del manejo de la aplicación radica en que el uso del dispositivo disminuiría el tiempo de ingreso de datos, además de asegurar que el descubrimiento de nueva información sea la correcta a partir de los datos de los que dispone online.

\section{TELECOMUNICACIONES Y SU REFORMA. Ecuador.}

10. Couch, L. (2008). Sistemas de comunicación digitales y analógicos. México: Pearson Educación.

11. EMPRESA PÚBLICA MUNICIPAL DE AGUA POTABLE. (s.f.). Obtenido de http://www.emapal.gob.ec/index.php?opt ion $=$ com_content $\&$ view $=$ article $\&$ id $=88 \&$ Itemid $=58$

12. Gómez, P. J. (31 de enero de 2011). Análisis de desempeño del nivel. Sistemas inalámbricos y móviles.

13. Harvey, Gordon. (2001). Cómo se citan las fuentes. Nuer, Madrid.

14. http://isa.uniovi.es/docencia/SIGC/pdf/tel efonia_movil.pdf

15. José Barceló Ordinas. (marzo de 2004). Redes de computadoras. España.

16. Naj, N. A. (5 de junio de 2013). Attribution Non-commercial. Recuperado el 2 de marzo de 2014, de es.scribd.com: http://es.scribd.com/doc/145791290/Mar coTeorico\#scribd

17. Secretaría Nacional de Planificación y Desarrollo (2013), Plan Nacional del Buen Vivir 2013 -2017, Quito.

18. Umberto. (2001). Cómo se hace una TESIS. Técnicas y procedimientos de estudio, investigación y escritura. Gedisa, Barcelona. 